\title{
Miotic Action of Tramadol is Determined by CYP2D6 Genotype
}

\author{
O. SLANA $\check{R}^{1,2}$, M. NOBILIS ${ }^{3}$, J. KVĚTINA ${ }^{3}$, R. MIKOVINY ${ }^{1}$, T. ZIMA ${ }^{2}$, \\ J. R. IDLE ${ }^{1}$, F. PERLÍK ${ }^{1}$
}

${ }^{I}$ Clinical Pharmacology Unit, Institute of Pharmacology and ${ }^{2}$ Institute of Clinical Biochemistry and Laboratory Diagnostics, First Faculty of Medicine, Charles University, Prague, ${ }^{3}$ Institute of Experimental Biopharmaceutics, Joint Research Centre of Academy of Sciences of the Czech Republic and PRO.MED.CS Praha a.s., Hradec Králové, Czech Republic

Received October 4, 2005

Accepted February 18, 2006

On-line available February 23, 2006

\begin{abstract}
Summary
Polymorphic CYP2D6 is the enzyme that activates the opioid analgesic tramadol by O-demethylation to its active metabolite O-demethyltramadol (M1). Our objective was to determine the opioid effects measured by pupillary response to tramadol of CYP2D6 genotyped volunteers in relation to the disposition of tramadol and M1 in plasma. Tramadol displayed phenotypic pharmacokinetics and it was possible to identify poor metabolizers (PM) with $>99 \%$ confidence from the metabolic ratio (MR) in a single blood sample taken between 2.5 and $24 \mathrm{~h}$ post-dose. Homozygous extensive metabolizers (EM) differed from $\mathrm{PM}$ subjects by an almost threefold greater $(\mathrm{P}=0.0014)$ maximal pupillary constriction $\left(\mathrm{E}_{\max }\right)$. Significant correlations between the AUC and $\mathrm{C}_{\max }$ values of $\mathrm{M} 1$ versus pupillary constriction were found. The corresponding correlations of pharmacokinetic parameters for tramadol itself were weaker and negative. The strongest correlations were for the single-point metabolic ratios at all sampling intervals versus the effects, with $r_{s}$ ranging from 0.85 to 0.89 (p $<0.01$ ). It is concluded that the concept of dual opioid/non-opioid action of the drug, though considerably stronger in EMs, is valid for both EM and PM subjects. This is the theoretical basis for the frequent use and satisfactory efficacy of tramadol in clinical practice when given to genetically non-selected population.
\end{abstract}

\section{Key words}

Cytochrome P450 2D6 • Miosis • Pharmacokinetics • Tramadol • Genotype

\section{Introduction}

Tramadol (2-[(dimethylamino)methyl]-1-(3methoxy-phenyl)cyclohexanol hydrochloride) is an orally-active and centrally-acting opioid analgesic used for the treatment of moderate to severe pain (Scott and Perry 2000). The marketed drug is the $( \pm)$-racemate derived from (1R,2R)-(+)-trans-tramadol and (1S,2S)-(-)trans-tramadol (Hui-Chen et al. 2004). Tramadol owes its pharmacological activity to the fact that it is biotransformed to the active metabolite O-demethyltramadol (M1) in the liver (Paar et al. 1992, Wu et al. 2002). Studies using the cloned human $\mu$-opioid receptor have established that (+)-M1 has 200-times higher 
affinity than the parent ( \pm )-tramadol (Gillen et al. 2000). Moreover, $(+)-M 1$ had the greatest intrinsic efficacy in an in vitro screen (Gillen et al. 2000).

In addition, there is an evidence that non-opioid mechanisms are involved in the analgesic properties of tramadol, in particular the inhibition of neuronal reuptake of both noradrenaline and 5-HT. In this regard, $(-)$-tramadol and $(+)$-tramadol are potent inhibitors of noradrenaline and 5-HT reuptake, respectively (Scott and Perry 2000). However, (-)-M1 is also a potent monoamine reuptake inhibitor (Garrido et al. 2000) and it potentiates the antinociceptive effects of (+)-M1 in rats (Garrido et al. 2000). Both opioid and non-opioid mechanisms are thought to act synergistically in the CNS (Garrido et al. 2000, Scott and Perry 2000).

CYP2D6 is a cytochrome P450 that is expressed in human liver, kidney, intestine and brain (Miksys et al. 2005), displays genetic polymorphism (Mahgoub et al. 1977) and metabolizes a wide range of drugs and endogenous neurochemicals (Yu et al. 2004). Both (+)and (-)-tramadol were first shown to be O-demethylated by CYP2D6 to their corresponding M1 metabolites by Dengler and his colleagues (Paar et al. 1992). Later other authors confirmed the importance of both CYP2D6 activity and CYP2D6 genotype for the pharmacokinetics of tramadol and M1 (Borlak et al. 2003, Gan et al. 2002, Levo et al. 2003). A negative impact of functionally deficient variants of CYP2D6 on the analgesic action of the drug was described in an experimental pain model in healthy volunteers (Poulsen et al. 1996), as well as in patients undergoing abdominal surgery (Stamer et al. 2003). Studies in rats have also linked the antinociceptive effects of intravenous (+)-tramadol to its conversion to (+)-M1 by CYP2D enzymes (Garrido et al. 2003).

Infrared pupillography is a well established method to determine the opioid mechanism of action in vivo. The regulation of pupillary diameter is a complex process for maintaining the dynamic balance in the tone of two key muscles, $\mathrm{m}$. dilatator pupillae and $\mathrm{m}$. sphincter pupillae. The sphincter is innervated by parasympathetic neurons, whereas the dilatator is stimulated by sympathetic nerves originating in the hypothalamus. The tone of the sphincter has greater impact on the final pupillary diameter and is regulated at the central level by increased stimulation of the Edinger-Westfal nucleus, which is the site where the miotic effect of $\mu$-agonists is generated. Morphine, a model example of such a compound, induces deep miosis when administered systematically, but almost no miotic effect is induced after local administration of high doses to the eye (Thompson 1987). On the other hand, compounds causing the blockade of noradrenaline reuptake are known to stimulate the dilatation of pupillae through indirect stimulation of $\alpha$-adrenergic receptors and an increase in the sympathetic tone. Therefore, it is expected that the effect of tramadol and M1 on pupillary diameter will be antagonistic. The parent compound itself generates mydriatic reaction, whereas M1 induces pupillary constriction via central stimulation of $\mu$-opioid receptors. A number of authors have reported that tramadol elicits miosis (Collart et al. 1993, Freye and Latasch 2000, Knaggs et al. 2004, Krueger and MüllerLimmroth 1978), while others have reported conflicting findings (Preston et al. 1991). The discrepancy of the results could be a result of polymorphic CYP2D6 pathway and thus phenotype-dependent pharmacokinetics of tramadol and in the formation of M1.

Due to the complexity of pharmacodynamic and pharmacokinetic features of tramadol, the real contribution of opioid and non-opioid mechanisms of action to the efficacy in human remains poorly understood.

Pain is one of the most common symptoms in clinical practice, although the complexity of its mechanisms is still poorly understood. Since there are many diverse confounding factors related to social, cognitive, psychological, or general health aspects influencing the patients' evaluation of pain relief, several experimental pain models have been designed to evaluate the drug efficacy of analgesics in healthy volunteers (Staahl and Drewes 2004). The experimental pain models still have substantial shortcomings mainly in nonspecificity of stimuli applied and thus weak ability to determine the pain/analgesic mechanisms involved. Though very complex, pupillar reactions and their regulatory mechanisms are reasonably well described (Thompson 1987), and therefore pupillography is one of a useful pharmacological tools to assess drug actions and their mechanisms in vivo in human subjects (Böttcher 1999). Our study was therefore designed to determine the miotic action of the drug by pupillography as a measure of opioid action in relation to the pharmacokinetics of both parent compound and M1 in three distinct groups of healthy volunteers characterized as CYP2D6 homozygous extensive metabolizers (homozygous EMs), heterozygous extensive metabolizers (heterozygous EMs) and poor metabolizers (PMs). 
Table 1. Demographic characteristics of subjects enrolled in the study.

\begin{tabular}{llll}
\hline & $\begin{array}{l}\text { Homozygous EMs } \\
(\mathbf{n}=7)\end{array}$ & $\begin{array}{l}\text { Heterozygous EMs } \\
(\mathbf{n}=7)\end{array}$ & $\begin{array}{l}\text { PMs } \\
(\mathbf{n}=7)\end{array}$ \\
\hline Genotype status & $* 1 /{ }^{*} 1(\mathrm{n}=7)$ & ${ }^{*} 1 /{ }^{*} 3(\mathrm{n}=2)$ & $* 3 /{ }^{*} 3(\mathrm{n}=1)$ \\
& & ${ }^{*} 1 /{ }^{*} 4(\mathrm{n}=5)$ & ${ }^{*} 3 / 4(\mathrm{n}=1)$ \\
Male/female ratio & $4 / 3$ & & $4 / 44(\mathrm{n}=5)$ \\
Age (years) & $21.7 \pm 0.5$ & $2 / 5$ & $3 / 4$ \\
Smokers & 0 & $22.1 \pm 1.2$ & $23.1 \pm 3.1$ \\
\hline
\end{tabular}

The values are presented as the mean \pm SD.

\section{Methods}

\section{Volunteer recruitment and CYP2D6 genotyping}

Twenty-one volunteers were selected from a database of 270 healthy medical students that had previously been genotyped for the presence of CYP2D6*3, *4, *5, *6 alleles and gene duplications using a simplified method by Sachse et al. (1997). Subjects, who did not possess one of the common inactivating alleles or gene duplications, were taken to be homozygous EMs, carriers of one variant allele were considered to be heterozygous EMs, and carriers of two variant alleles were classified as PMs. A full medical history was taken from each volunteer, Table 1 shows basic demographic description of the subjects. All subjects were required to abstain from taking any drugs for a period of four weeks prior to the study and alcohol for three days prior to the study. Smokers agreed to smoke less than ten cigarettes per day for 10 days prior to the study. The volunteers gave their written informed consent before participating and the study protocol had been approved by the Ethics Committee of the First Faculty of Medicine and General Teaching Hospital, Prague.

\section{Drug administration and pharmacokinetics}

Each genotyped volunteer was administered orally a slow-release tablet containing $100 \mathrm{mg}$ of tramadol hydrochloride (Tramal ${ }^{\circledR}$ Retard 100, Léčiva Praha a.s., Czech Republic). Blood samples were taken into heparinized syringes $(9 \mathrm{ml}$, Sarstedt S-Monovette, Sarstedt, Germany) before drug administration and 2.5, 4, 8,12 , and $24 \mathrm{~h}$ post-dose. Between samplings, cannulae were flushed with heparinized saline $(10 \mathrm{U} / \mathrm{ml}$; Léčiva Praha a.s., Czech Republic). After sampling at $12 \mathrm{~h}$, the cannulae were removed and the final $24 \mathrm{~h}$ sample was taken by venepuncture. Plasma was obtained by centrifugation at $3000 \mathrm{~g}$ and the specimens were then stored within $30 \mathrm{~min}$ at $-20^{\circ} \mathrm{C}$ until analysis. Tramadol and $\mathrm{M} 1$ concentrations in plasma (nM) were determined by fluorescence HPLC (Nobilis et al. 2002). Pharmacokinetic parameters were derived by noncompartmental analysis using WinNonlin version 4.1 (Pharsight Corporation, Mountain View CA, USA) running on MS Windows 2000 SP4.

\section{Pupillography}

Dark-adapted horizontal and vertical pupillary diameters in both eyes were determined by infrared pupillography, before drug administration and at 2.5, 4, 8, and $12 \mathrm{~h}$ post-dose. Prior to the study, volunteers were thoroughly informed about the procedures and were allowed to become familiar with the study rooms. For all time intervals, the sequence of interventions was blood sampling from an indwelling cannula followed by pupillography in a quiet, darkened and well-heated room.

We used a simple method to measure darkadapted pupillary diameter by using a commercially available digital camera with a supplemental infrared mode of recording (Sony DSC-F707; 5.0 megapixel). The method was validated previously (Slanař et al. 2005). The volunteers, with a flat calibrated standard of $30 \mathrm{~mm}$ length, which was attached to the cheek under the eye, were seated in a dark room and, after 5 min of dark adaptation, three infrared photographs of both eyes were taken. The diameter of pupil on each photograph was measured in pixels using the measurement tool of Adobe Photoshop 6.0 software, as well as the length of the standard. The actual size of the pupil was then calculated according to the formula $\mathrm{D}_{\mathrm{mm}}=30 / \mathrm{S}_{\mathrm{pix}} * \mathrm{D}_{\text {pix }}$, where $\mathrm{D}_{\mathrm{mm}}$ and $\mathrm{D}_{\text {pix }}$ represent pupillary diameter in $\mathrm{mm}$ and pixels, respectively, and $\mathrm{S}_{\text {pix }}$ the length of the standard in pixels. 


\section{a: $(R, S)-( \pm)$-tramadol}

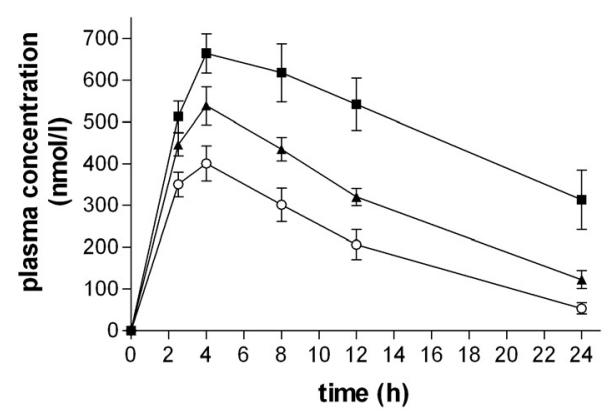

b: (士)-O-demethyltramadol

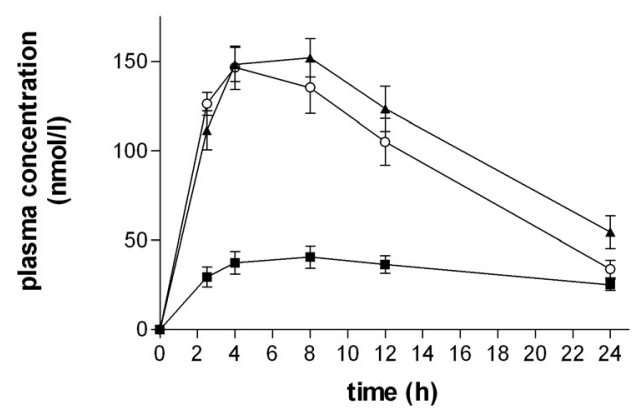

c: effect

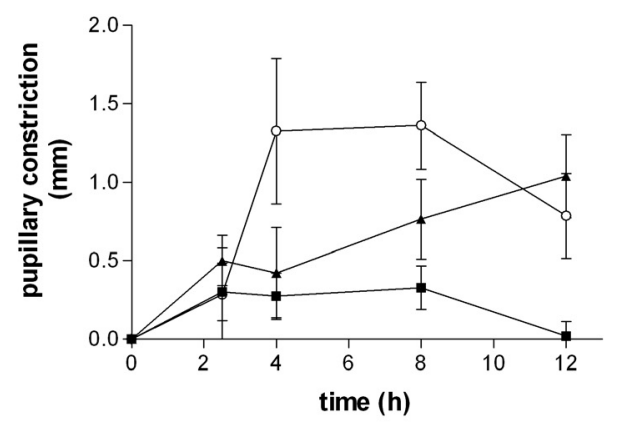

Fig. 1. Plasma concentration-time profiles of a $(R, S)-( \pm)-$ tramadol and $\mathbf{b}( \pm)$-O-demethyltramadol as well as $\mathbf{c}$ pupillary constriction (basal diameter - diameter at time t) after a single dose of $100 \mathrm{mg}$ oral slow release racemic tramadol for homozygous extensive metabolizers (open circles), heterozygous extensive metabolizers (open triangles), and poor metabolizers (solid squares) of CYP2D6. Data are mean \pm SEM.

Both horizontal and vertical pupillary diameters in each photograph were estimated and used to calculate the mean of the three subsequent measurements, which was considered as the pupillary diameter, thus reducing the effect of physiological oscillations in pupil size. The eye with the larger basal dark-adapted diameter was selected and subsequent measurements were done in that eye.

\section{Statistical methods}

Descriptive statistics (mean $\pm \mathrm{SD}$ ) was used to present the data and one-way analysis of variance (ANOVA) was applied to evaluate the intergenotype differences. Statistical evaluations were performed using Statistica for Windows version 6.0 (StatSoft, USA).

\section{Results}

Figure 1 shows the plasma concentration-time profiles of tramadol and M1 and the effect-time profile in the three genotype groups. The concentrations of tramadol and M1 in PM group are substantially different from both EM groups. The tramadol plasma levels in PMs were found at all sampling intervals higher than in the other two genotype groups, while the lowest mean concentrations have been observed in the homozygous EM subjects. The $\mathrm{C}_{\max }$ levels in PMs were approximately $75 \%$ higher than in homozygous EMs, and the difference had been increasing over the sampling interval to approximately 4-fold variation between these two groups at $24 \mathrm{~h}$ post-dose. The concentrations of the parent compound in heterozygous EMs were approximately 30 \% higher than in homozygous EMs and about 30-70\% lower than in PM subjects. As could be expected, the production of M1 and subsequently the plasma levels of this compound have been substantially decreased in PMs with the $\mathrm{C}_{\max }$ approximately 5-times lower than in both EM groups. The M1 concentrations in both EM groups were not as much different, but the M1 plasma concentration-time curve in heterozygous EM subjects was slightly shifted into the right, probably as a result of decreased and delayed formation of M1. The effect as determined by mean pupillary constriction has been highest in homozygous EM subjects and lowest in PM group. The difference in mean pupillary constriction between these two groups was approximately 3-fold starting from $4 \mathrm{~h}$ post-dose. The pupillary constriction of heterozygous EM subjects increased over time with the maximal response to the drug at the end of followed time interval of $12 \mathrm{~h}$.

Pharmacokinetic parameters in the respective genotype groups are summarized in Table 2. Mean AUC and $\mathrm{C}_{\max }$ values of tramadol were substantially higher in PM group, while they were three- to four-times lower in case of M1 in the PM group when compared to both EM groups. All the parameters, $\mathrm{C}_{\max }, \mathrm{AUC}_{(0-24)}$ and $\mathrm{t}_{1 / 2}$, of either tramadol or M1, differed significantly between PM and homozygous EM or EM, subjects. 
Table 2. Pharmacokinetic parameters of $(R, S)-( \pm)$-tramadol and $( \pm)$-O-demethyltramadol $\left(M_{1}\right.$ metabolite) for three CYP2D6 genotype groups.

$$
\mathrm{C}_{\max }(\mathrm{nmol}) \quad \mathbf{t}_{\max }(\mathrm{h}) \quad \text { Half-life (h) } \quad \mathrm{AUC}_{0-24}(\mathrm{nmol} \times \mathrm{h} / \mathrm{l})
$$

\section{$(R, S)-( \pm)-$ tramadol}

Homozygous EMs

Heterozygous EMs

$\begin{array}{llll}413 \pm 101^{\mathrm{a}} & 4 & 6.31 \pm 0.94^{\mathrm{a}} & 4986 \pm 1763^{\mathrm{a}} \\ 547 \pm 116^{\mathrm{a}} & 8 & 9.23 \pm 2.90^{\mathrm{a}} & 7408 \pm 1266^{\mathrm{a}} \\ 699 \pm 155 & 8 & 25.89 \pm 11.88 & 11544 \pm 3121\end{array}$

$P M s$

$699 \pm 15$

$\begin{array}{lllll}\text { Homozygous EMS } & 151 \pm 32^{\mathrm{a}} & 4 & 7.87 \pm 1.12^{\mathrm{a}} & 2382 \pm 808^{\mathrm{a}} \\ \text { Heterozygous EMS } & 158 \pm 28^{\mathrm{a}} & 4 & 10.71 \pm 2.79^{\mathrm{a}} & 2553 \pm 567^{\mathrm{a}} \\ P M S & 41 \pm 16 & 4 & 24.96 \pm 7.28 & 768 \pm 280\end{array}$

$7.87 \pm 1.12^{\mathrm{a}}$

$2382 \pm 808^{\mathrm{a}}$

The values are presented as the mean $\pm S D$ and median for $t_{\max } . C_{\max }$ maximum plasma concentration, $t_{\max }$ time to reach $C_{\max }, A U C_{0-24}$ area under the plasma concentration-time curve from $0-24 \mathrm{~h}$ post-dose. ${ }^{\mathrm{a}} \mathrm{p}<0.05$ compared with the PM group.

Table 3. Pupillary response for three CYP2D6 genotype groups.

\begin{tabular}{llll}
\hline & Homozygous EMs & Heterozygous EMs & PMs \\
\hline$E_{\max }(\Delta m m)$ & $1.83 \pm 0.95^{\mathrm{a}}$ & $1.25 \pm 0.73$ & $0.58 \pm 0.24$ \\
$t_{\max }(h)$ & $5.71 \pm 2.14$ & $9.14 \pm 3.02$ & $5.64 \pm 3.74$ \\
$A U D_{0-12}(m m x h)$ & $12.14 \pm 6.85^{\mathrm{a}}$ & $7.31 \pm 4.94$ & $2.64 \pm 3.20$ \\
\hline
\end{tabular}

The values are presented as the mean $\pm S D$. $E_{\max }$ maximum pupillary response, $t_{\max }$ time to reach $E_{\max }, A D_{0-12}$ area under the pupillary response-time curve from $0-12 \mathrm{~h}$ post dose. ${ }^{a} \mathrm{p}<0.05$ compared with the PM group.

Table 4. Correlations (Spearman's test) of different pharmacokinetic parameters of $(R, S)$-( \pm )-tramadol and $( \pm)$-O-demethyltramadol ( $M_{1}$ metabolite) with pupillary response in 21 healthy volunteers.
$\mathbf{C}_{\max }(\mathbf{n m o l})$
Half-life (h)
$\operatorname{AUD}_{0-12}(\mathbf{m m} \times \mathbf{h})$

\section{(R,S)-(士)-tramadol}

$\begin{array}{llll}E_{\max }(\Delta \mathrm{mm}) & -0.39 * & -0.43 & -0.51^{*} \\ A U C_{0-24}(\operatorname{mmol} x \mathrm{~h} / \mathrm{l}) & -0.40 & -0.40 & -0.41^{*}\end{array}$

\section{(土)-O-demethyltramadol}

$\begin{array}{llll}E_{\max }(\Delta \mathrm{mm}) & 0.59^{* *} & -0.49 * & -0.55^{* *} \\ A U C_{0-24}(\mathrm{mmol} x \mathrm{~h} / \mathrm{l}) & 0.55^{* *} & 0.47^{*} & 0.52^{* *}\end{array}$

$\mathrm{C}_{\max }$ maximum plasma concentration, $\mathrm{AUC}_{0-24}$ area under the plasma concentration-time curve from 0-24 $\mathrm{h}, \mathrm{E}_{\max }$ maximum pupillary response, $A_{U D_{0-12}}$ area under the pupillary response-time curve from 0-12 h. Significance of correlation coefficients: ${ }^{*} p<0.05,{ }^{* *} p<0.01$

Tramadol AUC metabolic ratio [tramadol $\left.\mathrm{AUC}_{(0-24)} / \mathrm{M} 1 \mathrm{AUC}_{(0-24)}\right]$ in the homozygous EM, heterozygous EM and PM subjects were 2.23 \pm 0.52 , $2.98 \pm 0.66$ and $16.8 \pm 6.6$, respectively. Inter-genotype differences were significant between PM and both EM groups. Similarly, at all blood sampling times between
2.5 and $24 \mathrm{~h}$, there was a highly significant difference ( $\mathrm{P}$ values ranging from 0.0006 to 0.011 ) between the single-point plasma concentration tramadol metabolic ratio values for the PMs and EMs allowing to identify PM subjects with $>99 \%$ confidence. 
Drug-induced miosis was observed in all the three genotype groups. The small effect in the PM group was, however, consistent over time (Fig. 1). The time to maximum pupillary constriction $\left(\mathrm{T}_{\max }\right)$, maximum pupillary constriction effect $\left(\mathrm{E}_{\max }\right)$ and area under the time-effect concentration profile (AUD) values are shown in Table 3. The AUD and $E_{\max }$ values in homozygous EMs were significantly greater than the respective PM values.

Interestingly, we have found different patterns of miotic response, not only between PM and EM groups, but also in the pharmacokinetic parameters between heterozygous and homozygous EMs. The median time to maximal miosis was $4 \mathrm{~h}$ in the homozygous Ems, while it was $8 \mathrm{~h}$ in the heterozygous group, probably reflecting the later formation of M1 in these subjects.

The relationship between the pharmacokinetic parameters of tramadol and M1 versus pupillary constriction is summarized in Table 4. The correlations of pharmacokinetic parameters of M1 versus pupillary effect were stronger and positive in comparison with the respective values for the parent compound that were negative. However, we found the strongest correlation of the single-point metabolic ratio (plasma concentration of tramadol/plasma concentration of M1) at all sampling intervals (2.5-24 $\mathrm{h}$ post-dose) versus the effects, with range of $\mathrm{r}_{\mathrm{s}}$ from 0.85 to $0.89(\mathrm{p}<0.01)$.

\section{Discussion}

We report here that after a single administration of slow-release tramadol formulation the mean concentrations of tramadol and its metabolite M1 are significantly dependent upon CYP2D6 genotype. Our results are in agreement with previously published data (Poulsen et al. 1996). They do not only confirm previous data, but they also clarify important issues for the routine use of tramadol in clinical practice. Our observations confirm a genotype-dependent disposition of tramadol and M1 not only between EM and PM subjects, but also within the EM phenotype, discriminating the absence or presence of one inactivating CYP2D6 allele. To the best of our knowledge, no previously published studies have reported such a difference, mainly because other investigators have selected their subjects by phenotyping methods, which are incapable to distinguish heterozygous and homozygous EMs.

The pupillary constriction apparently does not correspond well with the tramadol or M1 plasma concentrations (Fig. 1). The discrepancy is pronounced mainly in heterozygous EM group, in which there are substantially high plasma levels of M1 until $12 \mathrm{~h}$ postdose, but the pupillary constriction raises during this interval until maximal effect at the end of followed time interval. Moreover, the mean effect at 2 and $4 \mathrm{~h}$ post-dose has been very small in heterozygous EMs. Also, the comparison between homozygous and heterozygous EM groups, which both have similar M1 concentrations, but substantially different effect-time profiles fails to demonstrate the correlation between observed pharmacokinetics and effect. This discrepancy may be caused by contradictory actions of the two main active compounds involved in the clinical efficacy of tramadol. While the miotic (opioid) action is believed to be mediated primarily by M1, the mydriatic effect can be exerted by the noradrenergic activity of the parent compound. Therefore the pupillary reaction cannot be attributed solely to plasma levels of either of these compounds, but it is a result of their combined effects. The different ratios of M1/tramadol plasma concentrations are likely to cause the apparent discrepancy in our results. We can speculate that there is a potential cut-point of metabolic ratio discriminating, whether significant miotic reaction will occur or not. However, our study does not allow to establish this cutpoint because it was not designed for such a purpose. Other factors like lag time of the effect vs. plasma concentrations may play a further role in modifying the comparison of concentration- vs. time-profiles of the drug.

Low, but detectable levels of M1 in PM group suggest that there must exist an alternative metabolic pathway converting tramadol to M1 except of CYP2D6. The M1 levels are also influenced by the activity of subsequent metabolic pathways because M1 is not the final metabolite and undergoes conjugation with sulphates and glucuronides. Further demethylation and hydroxylation of M1 produces some other metabolites (M5, M32) (Wu et al. 2002). If the metabolic pathways involved displayed functional polymorphisms, it could play a role not only in relation to the M1 plasma concentrations, but also as additional factors influencing the analgesic efficacy of the treatment.

It has been shown previously that the metabolism of both enantiomers of tramadol is dependent on the activity of CYP2D6 in humans, not only in the formation of $(+)-\mathrm{M} 1$, but also in the production of (-)-M1 (Poulsen et al. 1996). The plasma levels of (+)-M1 in PMs were hardly detectable and therefore the general conclusion was that the analgesic activity of the drug in 
PMs, which was considerably lower than in EMs, is mediated through the non-opioid monoaminergic pathways. However, we have observed a constant miotic action of this drug in PMs, suggesting a residual opioid mechanism of action, though considerably weaker than in both EM groups. The correlations of miotic action versus pharmacokinetic parameters of M1 were stronger in comparison with the correlations of the pharmacokinetics of parent compound to the miotic effect. Because (+)-M1 possesses approximately 70-times higher affinity to $\mu$ opioid receptors than (-)-M1 (Gillen et al. 2000), we can conclude that, even the very small amount of $(+)-\mathrm{M} 1$ that is produced in PMs, contributes to the opioid action of tramadol in this genotype. Even in these PM subjects, the miotic opioid effects of the drug are stronger than the mydriatic effects due to noradrenergic modulation by the parent compound. Thus the concept of a dual opioid/nonopioid drug action, though considerably weaker than in EMs, is also valid in the PM genotype.

We were not able to use a stereoselective analytical method to determine plasma levels of the drug and M1 metabolite. However, there is a considerable deficiency in the production of (+)-M1 in PMs, whereas approximately equivalent concentrations of both enantiomers of M1 and tramadol were found in EMs (Poulsen et al. 1996). Thus, the use of non-selective method is justified.

In agreement with the dependency of tramadol pharmacokinetics on CYP2D6 genotype, we could also discriminate two phenotypes on the basis of the miotic response. The $\mathrm{E}_{\max }$ of all PMs was $<1 \mathrm{~mm}$, whereas 9 of the 14 subjects representing phenotypic EM subjects responded to the single dose of tramadol with $\mathrm{E}_{\max }$ $>1 \mathrm{~mm}$. Substantial overlap of the miotic response in the
PM and EM subjects does not support to the use of this measurement as a reliable non-invasive phenotyping test after a single dose of tramadol. Large interindividual variability in pupillary diameter, due to the complexity of pupillary diameter regulation usually found in general population (Thompson 1987), could be due to a number of reasons. First, physiological pupillary oscillations occur with a slow frequency of $1 \mathrm{~Hz}$. To eliminate the influence of these oscillations on our results, three consecutive photographs were taken, and mean of these measurements was considered as a pupillary diameter. We used the same methodology that had previously been successfully validated with a Pupillscan II pupillometer. Second, variability in the opioid response might also be caused by recently described functional polymorphism of $\mu$-opioid receptor (Skarke et al. 2003), which we did not analyze in our population. However, This could be only an explanation for the variability in drug effects, not explaining the basal variations of pupillary diameter. Third, pupillary diameter largely depends on the age of the subject, which was eliminated by selection of the young-adult volunteers of comparable age.

It can be finally concluded that the observed opioid action of the drug in subjects of different CYP2D6 genotypes is a plausible explanation for the use of this drug in clinical practice. A dual mechanism of action, not only in EMs, but also in PMs, would provide a theoretical basis for the better efficacy of tramadol in PMs than should be expected from only the non-opioid effects of tramadol.

\section{Acknowledgements}

The work has been supported by a grant No. MSM0021620820.

\section{References}

BÖTTCHER M: Pupillography in clinical pharmacology. In: Pupillography: Principles, Methods and Applications. J KUHLMANN, M BÖTTCHER (eds), W. Zuckschwerdt Verlag, München 1999, pp 13-26.

BORLAK J, HERMANN R, ERB K, THUM T: A rapid and simple CYP2D6 genotyping assay--case study with the analgetic tramadol. Metabolism 52: 1439-1443, 2003.

COLLART L, LUTHY C, FAVARIO-CONSTANTIN C, DAYER P: Duality of the analgesic effect of tramadol in humans. (in French) Schweiz Med Wochenschr 123: 2241-2243, 1993.

FREYE E, LATASCH L: Effects of tramadol and tilidine/naloxone on oral-caecal transit and pupillary light reflex. Arzneimittelforschung 50: 24-30, 2000.

GAN SH, ISMAIL R, WAN ADNAN WA, WAN Z: Correlation of tramadol pharmacokinetics and CYP2D6*10 genotype in Malaysian subjects. J Pharm Biomed Anal 30: 189-195, 2002.

GARRIDO MJ, VALLE M, CAMPANERO MA, CALVO R, TROCONIZ IF: Modeling of the in vivo antinociceptive interaction between an opioid agonist, (+)-O-desmethyltramadol, and a monoamine reuptake inhibitor, (-)-Odesmethyltramadol, in rats. J Pharmacol Exp Ther 295: 352-359, 2000. 
GARRIDO MJ, SAYAR O, SEGURA C, RAPADO J, DIOS-VIEITEZ MC, RENEDO MJ, TROCONIZ IF: Pharmacokinetic/pharmacodynamic modeling of the antinociceptive effects of (+)-tramadol in the rat: role of cytochrome P450 2D activity. J Pharmacol Exp Ther 305: 710-718, 2003.

GILLEN C, HAURAND M, KOBELT DJ, WNENDT S: Affinity, potency and efficacy of tramadol and its metabolites at the cloned human mu-opioid receptor. Naunyn Schmiedebergs Arch Pharmacol 362: 116-121, 2000.

HUI-CHEN L, YANG Y, NA W, MING D, JIAN-FANG L, HONG-YUAN X: Pharmacokinetics of the enantiomers of trans-tramadol and its active metabolite, trans-O-demethyltramadol, in healthy male and female chinese volunteers. Chirality 16: 112-118, 2004.

KNAGGS RD, CRIGHTON IM, COBBY TF, FLETCHER AJ, HOBBS GJ: The pupillary effects of intravenous morphine, codeine, and tramadol in volunteers. Anesth Analg 99: 108-112, 2004.

KRUEGER H, MULLER-LIMMROTH W: Algo-pupillometric investigation of the analgesic effect of tramadol. (in German) Arzneimittelforschung 28: 176-178, 1978.

LEVO A, KOSKI A, OJANPERA I, VUORI E, SAJANTILA A: Post-mortem SNP analysis of CYP2D6 gene reveals correlation between genotype and opioid drug (tramadol) metabolite ratios in blood. Forensic Sci Int 135: 9-15, 2003.

MAHGOUB A, IDLE JR, DRING LG, LANCASTER R, SMITH RL: Polymorphic hydroxylation of debrisoquine in man. Lancet 2: 584-586, 1977.

MIKSYS S, CHEUNG C, GONZALEZ FJ, TYNDALE RF: Human CYP2D6 and mouse CYP2Ds: organ distribution in a humanized mouse model. Drug Metab Dispos 33: 1495-1502, 2005.

NOBILIS M, KOPECKÝ J, KVĚTINA J, CHLÁDEK J, SVOBODA Z, VOŘÍŠEK V, PERLÍK F, POUR M, KUNEŠ J: High-performance liquid chromatographic determination of tramadol and its O-desmethylated metabolite in blood plasma. Application to a bioequivalence study in humans. J Chromatogr A 949: 11-22, 2002.

PAAR WD, FRANKUS P, DENGLER HJ: The metabolism of tramadol by human liver microsomes. Clin Investig 70: 708-710, 1992.

POULSEN L, ARENDT-NIELSEN L, BROSEN K, SINDRUP SH: The hypoalgesic effect of tramadol in relation to CYP2D6. Clin Pharmacol Ther 60: 636-644, 1996.

PRESTON KL, JASINSKI DR, TESTA M: Abuse potential and pharmacological comparison of tramadol and morphine. Drug Alcohol Depend 27: 7-17, 1991.

SACHSE C, BROCKMOLLER J, BAUER S, ROOTS I: Cytochrome P450 2D6 variants in a Caucasian population: allele frequencies and phenotypic consequences. Am J Hum Genet 60: 284-295, 1997.

SCOTT LJ, PERRY CM: Tramadol: a review of its use in perioperative pain. Drugs 60: 139-176, 2000.

SKARKE C, DARIMONT J, SCHMIDT H, GEISSLINGER G, LOTSCH J: Analgesic effects of morphine and morphine-6-glucuronide in a transcutaneous electrical pain model in healthy volunteers. Clin Pharmacol Ther 73: 107-121, 2003.

SLANAŘ O, URBAN M, PERLÍK F: Infrared pupilometry measured by digital photography. (in Czech) Cas Lek Cesk 144: 273-276, 2005.

STAAHL C, DREWES A M: Experimental human pain models: a review of standardised methods for preclinical testing of analgesics. Basic Clin Pharmacol Toxicol 95: 97-111, 2004.

STAMER UM, LEHNEN K, HOTHKER F, BAYERER B, WOLF S, HOEFT A, STUBER F: Impact of CYP2D6 genotype on postoperative tramadol analgesia. Pain 105: 231-238, 2003.

THOMPSON HS: The pupil. In: Physiology of the Eye: Clinical Application. FH ADLER, RA MOSES (eds), Mosby, New York, 1981, pp 412-441.

WU WN, MCKOWN LA, LIAO S: Metabolism of the analgesic drug ULTRAM (tramadol hydrochloride) in humans: API-MS and MS/MS characterization of metabolites. Xenobiotica 32: 411-425, 2002.

YU AM, IDLE JR, GONZALEZ FJ: Polymorphic cytochrome P450 2D6: humanized mouse model and endogenous substrates. Drug Metab Rev 36: 243-277, 2004.

\section{Reprint requests}

Ondřej Slanař, Clinical Pharmacology Unit, Na Bojišti 1, 12000 Prague 2, Czech Republic, Fax: +420 224964133.

E-mail: oslan@lf1.cuni.cz 